\title{
Direct Measurement of the Flow Field around Swimming Microorganisms
}

\author{
Knut Drescher, Raymond E. Goldstein, Nicolas Michel, Marco Polin, and Idan Tuval \\ Department of Applied Mathematics and Theoretical Physics, University of Cambridge, Cambridge CB3 OWA, United Kingdom
}

(Received 3 May 2010; published 11 October 2010)

\begin{abstract}
Swimming microorganisms create flows that influence their mutual interactions and modify the rheology of their suspensions. While extensively studied theoretically, these flows have not been measured in detail around any freely-swimming microorganism. We report such measurements for the microphytes Volvox carteri and Chlamydomonas reinhardtii. The minute $(\sim 0.3 \%)$ density excess of $V$. carteri over water leads to a strongly dominant Stokeslet contribution, with the widely-assumed stresslet flow only a correction to the subleading source dipole term. This implies that suspensions of $V$. carteri have features similar to suspensions of sedimenting particles. The flow in the region around $C$. reinhardtii where significant hydrodynamic interaction is likely to occur differs qualitatively from a puller stresslet, and can be described by a simple three-Stokeslet model.
\end{abstract}

DOI: 10.1103/PhysRevLett.105.168101

PACS numbers: 87.17.Jj, 47.63.Gd, 87.16.Qp

Aided by advances in imaging techniques that allow detailed studies of the rotating flagella of bacteria [1] and the undulating flagella of spermatozoa [2] and algae [3], there is now a general consensus on how mechanical motions of microorganism appendages generate propulsive forces in a viscous fluid [4]. No such consensus exists yet on the origins of collective behavior [5], transport [6,7] and rheological properties of suspensions [8], and the interaction of organisms with surfaces $[9,10]$. As hydrodynamics surely plays a key role in these effects, a detailed knowledge of the flow field around freely swimming microorganisms is needed, both in the near field and far away. Here we present the first such measurements.

The linearity of the Stokes equations implies that the farfield flow around a microorganism can be expressed as a superposition of singularity solutions [11], with the slowest decaying mode dominating sufficiently far away. Theories of fluid-mediated interactions and collective behavior typically assume neutrally buoyant swimmers which exert no net force on the fluid. The thrust $T$ of their flagella and the viscous drag on their body are displaced a distance $d$ apart (often comparable to the cell radius $R$ ), and balance to give the far-field flow of a force dipole, or stresslet [12], which decays with distance $r$ as $T d / \eta r^{2}$, where $\eta$ is the fluid's viscosity. The contribution from a suspension of such stresslets to the fluid stress tensor is central to some of the most promising approaches to collective behavior of microorganisms [13].

The force-free idealization of swimmers requires precise density matching [9] not generally realized in nature. To appreciate the striking effects of gravity, one need only consider the buoyancy-driven plumes of bioconvection [14]. Models of this instability express the contribution of cells to the Navier-Stokes equations as a sum of force monopoles (Stokeslets), coarse grained as a body force proportional to the cell concentration and gravitational force $F_{g}$ per cell [14]. As the flow around a Stokeslet decays as $F_{g} / \eta r$, it is clear, if not appreciated previously, that there is a distance $\Lambda \sim T d / F_{g}$ at which the nearby stresslet contribution crosses over to the distant Stokeslet regime. This is one of several crossover lengths relevant to swimmers; for ciliates, unsteady effects become important on scales smaller than the viscous penetration depth [15]. For a given organism, the relevance of the length $\Lambda$ to a particular physical situation depends on the cell concentration and the observable of interest. At low concentrations the Stokeslet form suffices, but the near field is relevant to cell-cell interactions, especially in concentrated suspensions [16] and to tracer dynamics [7]. The notion of near field requires distinguishing between distances $r$ satisfying $R \ll r \ll \Lambda$, where a stresslet description may hold, and $r \sim R$ where the multipole contributions may not be well ordered and the flow topology can differ from that of a stresslet.

A synthesis of tracking microscopy and fluid velocimetry is used here to quantify the competing force singularities and the near-field flow topology for the unicellular biflagellate green alga Chlamydomonas reinhardtii [17] $(R \sim 5 \mu \mathrm{m})$ and its larger relative Volvox carteri [18], a spherical alga $(R \sim 200 \mu \mathrm{m})$ which swims by the action of $\sim 10^{3}$ Chlamydomonas-like cells on its surface. For Volvox our most significant finding is that the flow field is strongly dominated by its Stokeslet component, despite a density excess of a mere $\sim 0.3 \%$, much smaller than that of common unicellular organisms $(\sim 5 \%-10 \%)$. Moreover, the high symmetry of Volvox results in a leading near-field correction in the form of a source doublet, and a smaller stresslet. The flow around Chlamydomonas is compatible with a simple "puller" stresslet only at distances $\gtrsim 7 R$, where the fluid velocity is $\leqslant 1 \%$ of the swimming speed; closer to the cell, the flow topology reflects the finite separation of the flagellar and body forces.

V. carteri f. nagariensis (strain EVE) was grown axenically in standard Volvox medium (SVM) [19] with sterile 
air bubbling, whereas $C$. reinhardtii (strain UTEX 89) was grown axenically in tris-acetate-phosphate (TAP) medium [17] on an orbital shaker, both in a diurnal growth chamber with $16 \mathrm{~h}$ in artificial cool daylight ( $\sim 4000$ lux $)$ at $28^{\circ} \mathrm{C}$, and $8 \mathrm{~h}$ in the dark at $26^{\circ} \mathrm{C}$. The large difference in organism size between Volvox and Chlamydomonas required two distinct methods to measure the flows they create [20]. A CCD camera (Pike, Allied Vision Technologies) mounted on a continuously focusable microscope (Infinivar, Infinity Optics) and connected to a vertical motorized XY stage (Thorlabs) followed individual Volvox colonies as they swam upwards [21] in a straight line along the central axis of a $5 \times 5 \times 50 \mathrm{~mm}$ sample chamber filled with SVM at $21 \pm 1{ }^{\circ} \mathrm{C}$. The stage was controlled by a custom LabView routine. The fluid was seeded at volume fraction $10^{-5}$ with $1 \mu \mathrm{m}$ nile-red polystyrene microspheres (Invitrogen) illuminated by a vertical $\sim 500 \mu \mathrm{m}$ thick laser sheet $(\lambda=532 \mathrm{~nm})$. Volvox is phototactic [22] at this wavelength, and at the intensities used here it swims smoothly along the laser sheet. We recorded the flow field of 19 different colonies at $30 \mathrm{fps}$ for $\sim 2-3$ min each. The measured flow field $\mathbf{v}$ was obtained by particle image velocimetry (Dantec Dynamics). Background flows in the chamber were $<10 \mu \mathrm{m} / \mathrm{s}$.

We observed a dilute suspension $\left(\sim 3 \times 10^{6} \mathrm{cells} / \mathrm{cm}^{3}\right)$ of Chlamydomonas in TAP on a Nikon inverted microscope at $40 \times($ NA 0.6$)$ by exciting their chlorophyll autofluorescence with a laser $(635 \mathrm{~nm}, \sim 60 \mathrm{~mW})$, which also excited $1.6 \mu \mathrm{m}$ fluorescent polystyrene microspheres (FS04F, Bangs Labs) used as tracers. Cylindrical polydimethylsiloxane sample chambers $(5 \mathrm{~mm}$ radius, $0.4 \mathrm{~mm}$ height) were prepared, pacified, and filled following [7]. Experiments were performed at $21 \pm 1{ }^{\circ} \mathrm{C}$, with the laser providing the only light source. We focused on a plane $150 \mu \mathrm{m}$ inside the chamber to minimize surface effects, and recorded movies at $250 \mathrm{fps}$ (Fastcam SA3, Photron). Movies were analyzed with standard algorithms to track cells and tracers. For each cell swimming along the focal plane for more than $1 \mathrm{~s}(\sim 10$ body lengths), we collected the instantaneous velocity of all tracers at $r<14 R$, normalized by the swimmer's speed. The resulting $3.3 \times 10^{6}$ velocity vectors were binned into a $2.5 \mu \mathrm{m}$ square grid (shown in Fig. 4), and the mean of the well-resolved Gaussian in each bin was used for the flow field.

In both experiments $\mathbf{U}_{0}$ indicates the swimmer velocity, while $\mathbf{u}(\mathbf{r})$ and $\mathbf{v}(\mathbf{r})=\mathbf{u}(\mathbf{r})-\mathbf{U}_{0}$ are the velocity field in the laboratory and comoving frames, respectively.

A typical experimental flow field around Volvox is shown in Fig. 1(a). We fit these fields to a superposition of a uniform background velocity $\left(\mathbf{U}_{0}\right)$, a Stokeslet (St), a stresslet (str) and a source doublet (sd):

$$
\begin{aligned}
\mathbf{v}_{\mathrm{fit}}(\mathbf{r})= & -U_{0} \hat{\mathbf{y}}-\frac{A_{\mathrm{St}}}{r}(\mathbf{I}+\hat{\mathbf{r}} \hat{\mathbf{r}}) \cdot \hat{\mathbf{y}}-\frac{A_{\mathrm{str}}}{r^{2}}\left[1-3(y / r)^{2}\right] \hat{\mathbf{r}} \\
& -\frac{A_{\mathrm{sd}}}{r^{3}}\left(\frac{\mathbf{I}}{3}-\hat{\mathbf{r}} \hat{\mathbf{r}}\right) \cdot \hat{\mathbf{y}}
\end{aligned}
$$

where $\mathbf{I}$ is the unit tensor, $\hat{\mathbf{y}}$ is the upward vertical unit

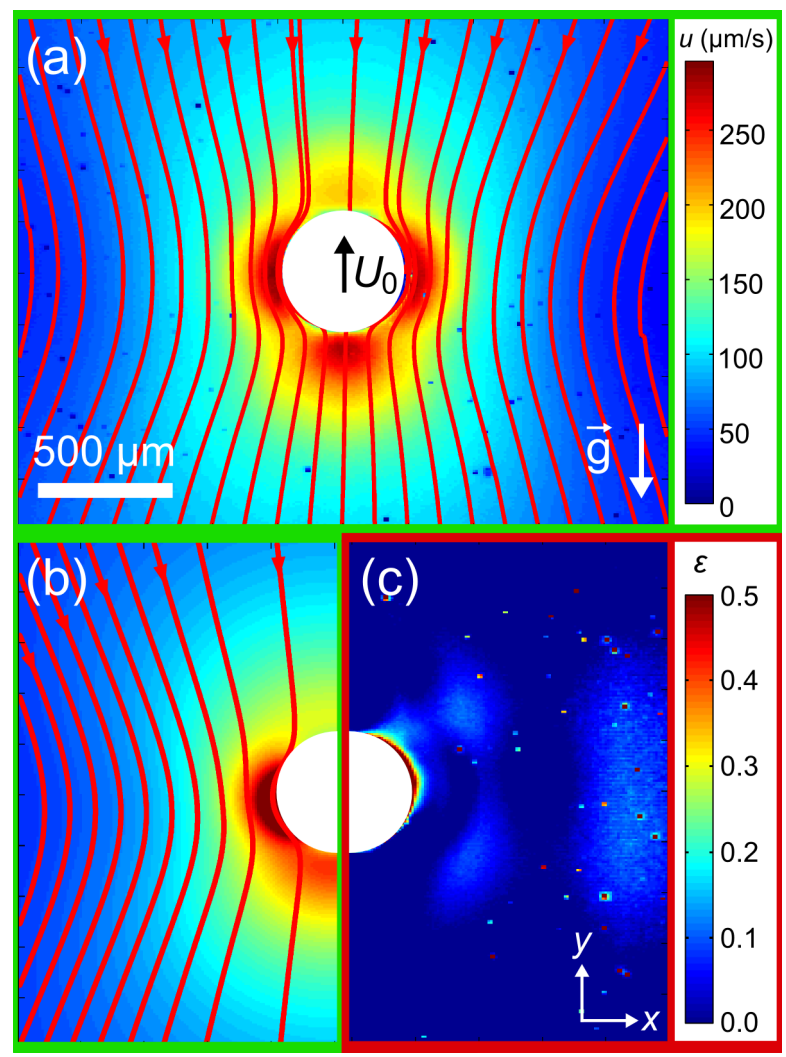

FIG. 1 (color online). Flow field of a freely swimming $V$. carteri in the laboratory frame. (a),(b) Magnitude and streamlines of $\mathbf{u}$ and its fitted approximation $\mathbf{u}_{\text {fit }}$, respectively. (c) Relative error of the fit: $\epsilon=\left\|\mathbf{u}-\mathbf{u}_{\text {fit }}\right\| /\|\mathbf{u}\|$. (a) and (b) have the same color bar, different from (c). $\vec{g}$ is gravity.

vector, $\hat{\mathbf{r}}=\mathbf{r} / r$, and $\mathbf{r}$ is measured from the center of the organism $\left(x_{c}, y_{c}\right)$. The orientation of all multipoles is fixed to be along the vertical, and we are left with six parameters: $\left(U_{0}, A_{\mathrm{St}}, A_{\mathrm{str}}, A_{\mathrm{sd}}, x_{c}, y_{c}\right)$. The fits, obtained by minimizing the integrated squared difference between the model and the experimental flows, describe remarkably well the experimental flow, almost down to the surface of the organisms [see Figs. 1(b) and 1(c)]. Typical values for the parameters are $U_{0} \sim 10^{2} \mu \mathrm{m} / \mathrm{s}, A_{\mathrm{St}} \sim 10^{4} \mu \mathrm{m}^{2} / \mathrm{s}, A_{\text {str }} \sim$ $10^{6} \mu \mathrm{m}^{3} / \mathrm{s}$ (indicating a pusher-type stresslet), $A_{\mathrm{sd}} \sim$ $10^{9} \mu \mathrm{m}^{4} / \mathrm{s}$, with the actual magnitude depending on the colony radius $R$. From the Stokeslet component, we can calculate the average colony density as $\Delta \rho=6 \eta A_{\mathrm{St}} / g R^{3}$, where $\eta=10^{-3}$ Pas and $g$ is the gravitational acceleration. The dependence of both $\Delta \rho$ and $U_{0}$ on $R$ (Fig. 2) compares well with previously published data [21] obtained by different means, thereby validating the measurements and analysis procedures. Removing the Stokeslet contribution from the experimental flow field [Fig. 3(a)] reveals that the near field is dominated by the source doublet component, with the stresslet responsible only for a slight forward-backward asymmetry [Figs. 3(b) and 3(c)]. The orientation of the source doublet is opposite to that around a translating solid sphere, and is compatible with a model that assigns a constant force density to the colony 


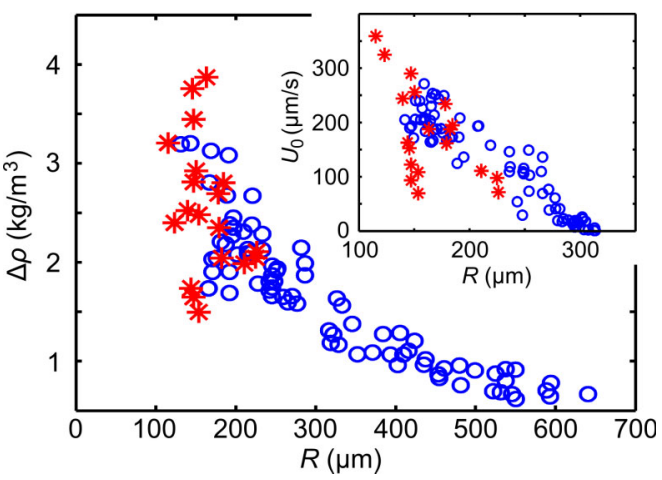

FIG. 2 (color online). The dependence of the excess density $\Delta \rho$ and swimming speed $U_{0}$ (inset) of $V$. carteri colonies (red stars) on their radius $R$ are compatible with previous measurements (blue circles) [21], and also [25].

surface [23], as well as a particular case of the "squirmer" model [24]. Average values of the parameters in Eq. (1) show that the crossover distance between the source doublet and stresslet $(\sim 3 R)$ is beyond that at which the Stokeslet becomes the leading component of the flow $(\sim 1.5 R)$. This peculiar ordering of multipoles results from the high degree of anterior-posterior symmetry of Volvox [22,23], and highlights the influence that a swimmer's body plan can have on its flow field.

The two $\sim 12 \mu \mathrm{m}$ long flagella of Chlamydomonas beat mostly in a synchronous breast stroke at $\sim 50 \mathrm{~Hz}$ [3], pulling the cell body through the fluid at speeds $U_{0} \sim$
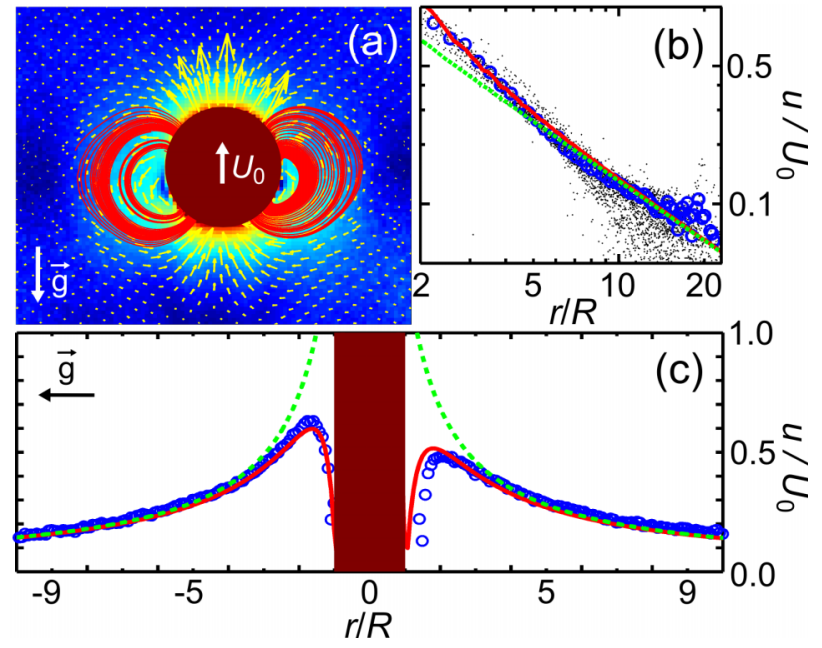

FIG. 3 (color online). Near field around V. carteri. (a) Magnitude, vector fields and streamlines of $\mathbf{u}$ after subtracting the fitted Stokeslet. Color bar as in Fig. 1(a). (b) $\|\mathbf{u}\|$ along a horizontal section through the center of the organism. The average Stokeslet (green dashed line) follows the decay of the experimental flow (blue circles) averaged over 19 different colonies (black dots). Deviations from a pure monopole appear from $\leqq 5 R$, and can be captured by adding a source doublet and a stresslet (red solid line). (c) Vertical section of the flow field $\mathbf{u}$ from the experiment in (a) through the center of the colony. The stresslet component is responsible for the forward-backward asymmetry. Symbols as in (b).
$100 \mu \mathrm{m} / \mathrm{s}$. Despite its $\sim 5 \%$ density excess over water [25], the gravitational Stokeslet of Chlamydomonas only becomes dominant at distances $\Lambda \geqslant 35 R$, as its ratio of $U_{0}$ to the sedimentation speed $\left(F_{g} / 6 \pi \eta R\right)$ is much larger than for Volvox. Therefore this swimmer has often been modeled as a puller stresslet [14]. A slight three-dimensionality of the beating causes the cell to spin about its swimming direction at $\sim 2 \mathrm{~Hz}$, so ensemble-averaged measurements of the kind presented here average out azimuthal asymmetries in the flow field. Figure 4(a) shows that for $r \geq 7 R$ the measured flow topology begins to resemble a puller stresslet, yet flow speeds at such distances are already $\leqslant 1 \mu \mathrm{m} / \mathrm{s}$. Closer to the organism, the field becomes more complex. It includes side vortices and a flow in front of the cell body that is along the direction of motion, towards a stagnation point. The velocity field can be accurately captured, even in the near field, by modeling the flow created by the pulled cell body as a Stokeslet, distributing the thrust among two Stokeslets located at the approximate positions of the two flagella, and averaging the flow over one rotation about $\mathbf{U}_{0}$. The streamlines of this simple extension to the force dipole model [see Fig. 4(b)], as well as the decay of $\|\mathbf{u}(\mathbf{r})\|$ with distance [see Fig. 4(c)], are very similar to those measured. Including no-slip boundary conditions on the cell body [26] has little effect on the velocity field as the cell-drag Stokeslet nearly produces the appropriate velocity field on the cell surface.

These flow field measurements around freely swimming microorganisms provide the basis for a deeper understanding of a number of issues in biological fluid dynamics, including the interactions of microorganisms with surfaces, with each other, and the rheology of suspensions. For example, it was recently discovered that Volvox colonies can form hydrodynamic bound states whose properties are quantitatively described by a model of interacting Stokeslets near a no-slip wall [21]. The near complete dominance of the flow field around Volvox by the Stokeslet term found here provides ex post facto justification for the neglect of higher moments. Perhaps more importantly this result shows that in terms of interparticle hydrodynamic couplings a suspension of Volvox is like a sedimenting suspension [27], except that the velocity of each colony is the sum of a self-propelled contribution and mutual advection in the flow field of other spheres. Elsewhere we illustrate this correspondence in detail [28].

The correspondence between the measured time- and azimuthally-averaged flow field of Chlamydomonas and the three-Stokeslet model illustrates how well such a simplification captures the complex flow topology, lending support to this approximation in modeling ciliary interactions [29]. Our results indicate that the simple puller-type description for Chlamydomonas is only valid at distances $\geq 7 R$, where the flow field is already $\lesssim 1 \%$ of $U_{0}$. We then expect interactions with other swimmers, boundaries or tracers, to be influenced mostly by the flow structure at shorter separations, where the full time dependence of the 

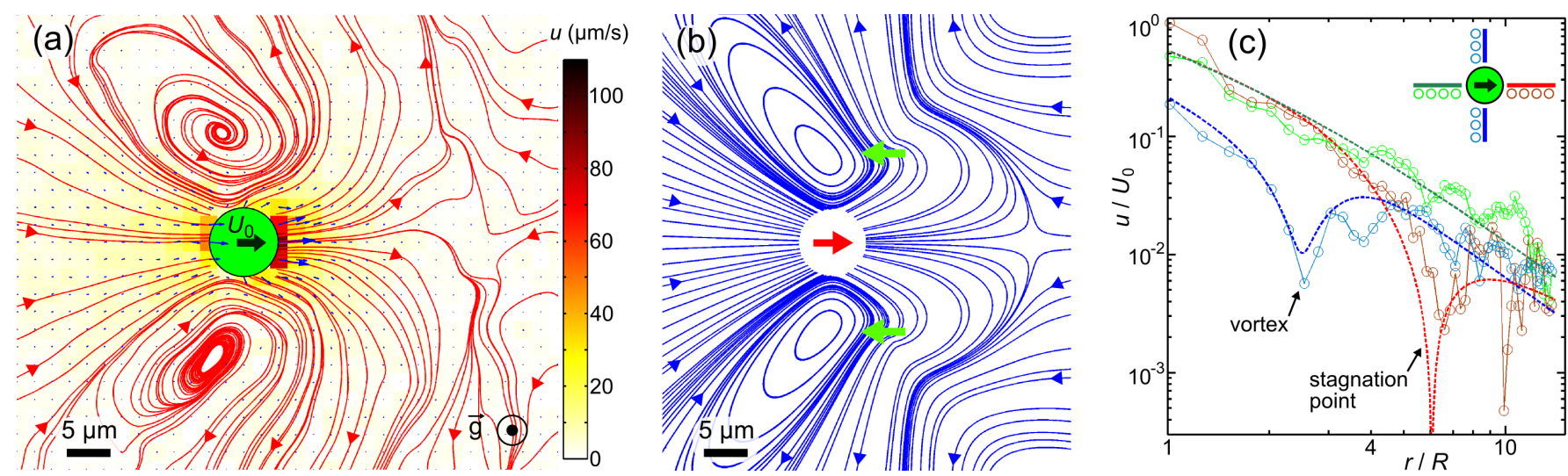

FIG. 4 (color online). Time- and azimuthally-averaged flow field of C. reinhardtii. (a) Streamlines (red [medium gray]) computed from velocity vectors (blue [dark gray]). The spiraling near elliptic points is an artifact of the direct integration of a noisy experimental velocity field. A color scheme indicates flow speed magnitudes. (b) Streamlines of the azimuthally-averaged flow of the three-Stokeslet model: flagellar thrust is distributed among two Stokeslets placed (not fitted) at the approximate flagellar position (lateral green arrows), whose sum balances drag on the cell body (central red arrow). (c) Decay of $\|\mathbf{u}(\mathbf{r})\|$ for the three directions indicated by separate colors in the inset, compared to results from the three-Stokeslet model (dashed lines).

flow may be important [30]. We are currently investigating whether similar conclusions hold for the flow field around bacteria, the prototypical "pusher" microorganisms.

We thank K. C. Leptos for suggesting the use of autofluorescence to track Chlamydomonas cells, S. B. Dalziel, V. Kantsler, and T. J. Pedley for discussions, D. Page-Croft and N. Price for technical assistance, and acknowledge support from the EPSRC, the BBSRC, the Marie-Curie Program (M.P.), and the Schlumberger Chair Fund.

[1] L. Turner, W. S. Ryu, and H. C. Berg, J. Bacteriol. 182, 2793 (2000).

[2] B. M. Friedrich, I.H. Riedel-Kruse, J. Howard, and F. Jülicher, J. Exp. Biol. 213, 1226 (2010).

[3] M. Polin et al., Science 325, 487 (2009).

[4] C. Brennen and H. Winet, Annu. Rev. Fluid Mech. 9, 339 (1977); E. Lauga, T. R. Powers, Rep. Prog. Phys. 72, 096601 (2009).

[5] C. Dombrowski et al., Phys. Rev. Lett. 93, 098103 (2004).

[6] P. T. Underhill, J. P. Hernandez-Ortiz, and M. D. Graham, Phys. Rev. Lett. 100, 248101 (2008).

[7] K. Leptos et al., Phys. Rev. Lett. 103, 198103 (2009).

[8] Y. Hatwalne et al., Phys. Rev. Lett. 92, 118101 (2004); T. Ishikawa and T. J. Pedley, J. Fluid Mech. 588, 399 (2007); A. W. C. Lau and T. C. Lubensky, Phys. Rev. E 80, 011917 (2009); A. Sokolov and I. S. Aranson, Phys. Rev. Lett. 103, 148101 (2009).

[9] A. P. Berke et al., Phys. Rev. Lett. 101, 038102 (2008).

[10] G. Li and J. X. Tang, Phys. Rev. Lett. 103, 078101 (2009); D. G. Crowdy and Y. Or, Phys. Rev. E 81, 036313 (2010).

[11] C. Pozrikidis, Boundary Integral and Singularity Methods for Linearized Viscous Flow (Cambridge University Press, Cambridge, 1992).
[12] G. K. Batchelor, J. Fluid Mech. 41, 545 (1970).

[13] R. A. Simha and S. Ramaswamy, Phys. Rev. Lett. 89, 058101 (2002); J.P. Hernandez-Ortiz, C. G. Stoltz, and M. D. Graham, Phys. Rev. Lett. 95, 204501 (2005); D. Saintillan and M. J. Shelley, Phys. Fluids 20, 123304 (2008); T. J. Pedley, J. Fluid Mech. 647, 335 (2010).

[14] T. J. Pedley and J. O. Kessler, Annu. Rev. Fluid Mech. 24, 313 (1992).

[15] C. Brennen, J. Fluid Mech. 65, 799 (1974).

[16] T. Ishikawa, M. P. Simmonds, and T. J. Pedley, J. Fluid Mech. 568, 119 (2006); C. M. Pooley, G. P. Alexander, and J. M. Yeomans, Phys. Rev. Lett. 99, 228103 (2007).

[17] E. H. Harris, The Chlamydomonas Sourcebook (Academic Press, Oxford, 2009), Vol. 1.

[18] D.L. Kirk, Volvox (Cambridge University Press, Cambridge, 1998).

[19] D. L. Kirk and M. M. Kirk, Dev. Biol. 96, 493 (1983).

[20] See supplementary material at http://link.aps.org/ supplemental/10.1103/PhysRevLett.105.168101 for movies.

[21] K. Drescher et al., Phys. Rev. Lett. 102, 168101 (2009).

[22] K. Drescher et al., Proc. Natl. Acad. Sci. U.S.A. 107, 11171 (2010).

[23] M. B. Short et al., Proc. Natl. Acad. Sci. U.S.A. 103, 8315 (2006).

[24] J. R. Blake, J. Fluid Mech. 46, 199 (1971).

[25] C. A. Solari et al., Am. Nat. 167, 537 (2006).

[26] C. W. Oseen, Hydrodynamik (Akadem. Verlagsgesellsch, Leipzig, 1927).

[27] R.E. Caflisch and J.H.C. Luke, Phys. Fluids 28, 759 (1985).

[28] I. Rushkin et al. (to be published).

[29] A. Vilfan and F. Jülicher, Phys. Rev. Lett. 96, 058102 (2006); T. Niedermayer, B. Eckhardt, and P. Lenz, Chaos 18, 037128 (2008).

[30] J.S. Guasto, K. A. Johnson, and J. P. Gollub, following Letter, Phys. Rev. Lett. 105, 168102 (2010). 